\title{
CARDIAC AND VASCULAR PATHOPHYSIOLOGY IN HYPERTENSION
}

Jamil Mayet, Alun Hughes

Heart 2003;89:1104-1109

$\square$ ypertension is one the earliest recorded medical conditions (Nei J in by Huang Ti around 2600BC); it has shaped the course of modern history ${ }^{1}$ and the consequences of hypertension (myocardial infarction, strokes, and heart failure) will soon be the leading global cause of death. Nevertheless, despite intensive research, the aetiology of hypertension remains obscure; only around $5 \%$ of cases have an identifiable cause. ${ }^{2}$ Indeed, primary or essential hypertension is perhaps better not considered a disease at all, ${ }^{\text {w1 }}$ rather (as suggested by Sir Geoffrey Rose) a level of blood pressure above which treatment does more good than harm. An individual's blood pressure depends on the complex interplay of heart and blood vessels and understanding this relation is the key to understanding the pathophysiology of hypertension.

\section{NORMAL CARDIAC AND VASCULAR PHYSIOLOGY}

Relation between mean pressure and mean flow in the human circulation The role of the circulation is to deliver blood to the tissues and flow occurs because of the pressure difference established by the pumping action of the heart. The relation between the pressure difference and flow can be described by a relation that is analogous to Ohm's Law for electrical current (box 1) and sometimes termed Darcy's Law.

This relation can be restated for the whole circulation in terms of mean arterial pressure, cardiac output, and peripheral resistance (box 2).

Although a simplification, this emphasises that an elevation of mean blood pressure can only come about as a result of an increase in cardiac output (CO), an increase in total peripheral vascular resistance (PVR), or a combination of both.

$\mathrm{CO}$ is a consequence of left ventricular pump function, which in turn depends on a number of factors (fig 1) including preload (venous return or left ventricular end diastolic volume (LVEDV)), the contractile state of the ventricular muscle, and afterload, an ill defined concept that is often equated with PVR in vivo, but is better characterised as the aortic input impedance, ${ }^{\text {w2 }}$ namely the complex relation between unsteady flow and pressure throughout the cardiac cycle.

PVR is dominated by small muscular arteries and arterioles (vessels with diameters between 30-300 $\mu \mathrm{m}) .{ }^{\mathrm{w} 3}$ Flow through an individual artery can be described by the Hagen-Poiseuille's equation (box 3).

So small changes in arterial diameter (or radius) have a profound effect on flow (or resistance). In the short term, the diameter of small arteries and arterioles is controlled by the contractile state of their smooth muscle. This in turn is regulated by a host of local and systemic factors ( fig 1 ).

Pulsatile pressure and flow in the circulation

The discussion above assumes that pressureand flow in the circulation are steady. This is clearly not the case. Cardiac output depends on the dynamic interaction between the mechanics of ejection and the properties of the vasculature. Pressure and flow show cyclical changes at all sites in the circulation, although the changes are considerably damped in capillaries. ${ }^{3}$ This damping is due mainly to the elastic behaviour of large elastic arteries, such as the aorta. and is termed the Windkessel or cushioning function (fig 2): in effect some of the pressure energy generated by the left ventricle is converted to elastic energy through distending large arteries. Once the heart ceases ejection and pressure falls, the walls of these arteries recoil and the elastic energy is reconverted into pressure. This reduces the magnitude of pressure change and accounts (to a large extent) for the diastolic component of arterial pressure.

Although this model is an oversimplification, it emphasises that arterial compliance (stiffness or elastance is the reciprocal of compliance) is an important factor in damping pressure oscillation and that pulse pressure will increase when arteries stiffen (for example, with aging). Moreover it also makes clear that changes in arterial stiffness per se should affect pulse pressure and not mean pressure. $^{4}$ w4

M ore detailed examination of the arterial pressure waveform in different segments of the arterial tree shows that it undergoes complex changes in shape ${ }^{5}$ that cannot be explained by the Windkessel model. Perhaps most surprising is that there is a consistent rise in systolic pressure (but not 


\section{Box 1}

$\Delta \mathrm{P}=\mathrm{Q} \times \mathrm{R}$

(where $\Delta \mathrm{P}=$ pressure difference. $\mathrm{Q}=$ bulk flow, $\mathrm{R}=$ resistance)

\section{Box 2}

\section{$M A P=C O \times P V R$}

(where MAP = mean arterial pressure, $C O=$ cardiac output (= stroke volume $\times$ heart rate), PVR $=$ total peripheral vascular resistance)

\section{Box 3}

\section{$Q=\pi r^{4} / 8 \eta L$}

(where $Q=$ flow, $\eta=$ fluid viscosity, $L=$ vessel length, $r=$ vessel radius)

mean pressure) in a peripheral artery such as the tibial artery or the brachial artery compared with the aorta. Generally this augmentation of systolic pressure is small $(\sim 7 \mathrm{~mm} \mathrm{Hg})$ and tends to diminish with age. But during exercise or in some young individuals at rest the difference between brachial and aortic pressures can exceed $30 \mathrm{~mm} \mathrm{Hg}$. In the latter case this

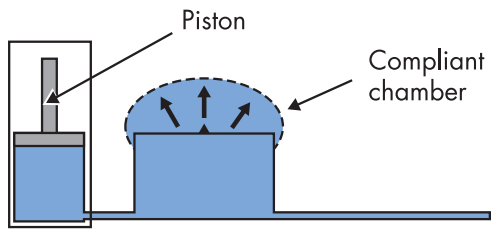

- The heart ejects intermittently into a compliant vessel(s) (aorta)

- Some of the pressure energy is converted into elastic energy in the wall; as pressure falls this is reconverted into pressure energy and damps the pressure change

- This can explain the damping but not the waveform change

Figure 2 The $\mathrm{W}$ indkessel model of the circulation. The circulation can be approximated by a pump connected to a compliant chamber (elastic arteries) and a flow resistance (resistance vasculature).

may give rise to spurious diagnoses of hypertension. ${ }^{\text {w5 }}$ This augmentation is believed to be caused by reflection of pressure waves. Reflected pressure waves are clinically important since they may place an additional load on the heart and vasculature (for example, in hypertension or heart failure). When the heart contracts it emits a forward travelling wave of pressure (technically termed a forward compression wave as it is a "blowing" as opposed to a "sucking" wave). ${ }^{6}$ This pressure wave travels rapidly through the circulation at speeds of $\sim 10 \mathrm{~m} / \mathrm{s}$ and is responsible for the palpable pulse. The speed of

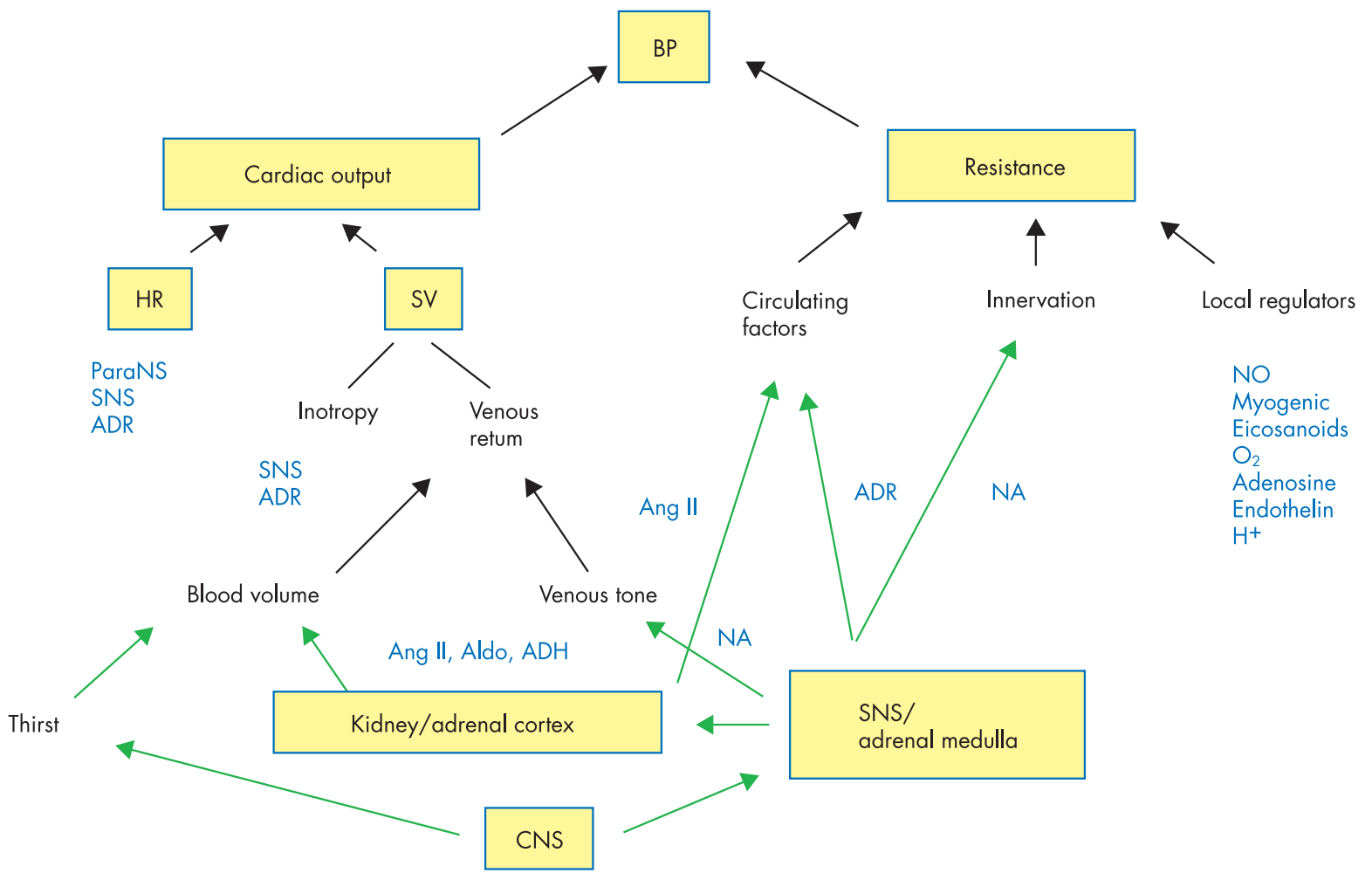

Figure 1 Mechanisms regulating mean arterial blood pressure. ADR, adrenaline; Aldo, aldosterone; Ang II, angiotensin II; ADH, antidiuretic hormone (arginine vasopressin); BP, blood pressure; CN S, central nervous system; HR, heart rate; N O, nitric oxide; ParaN S, parasympathetic nervous system; SV, stroke volume; SN S, sympathetic nervous system. 
Box 4: Haemodynamic changes in established

essential hypertension

$\uparrow \mathrm{PVR}$

$\uparrow$ central shift in blood volume

$\rightarrow$ cardiac index, stroke volume, extracellular fluid volume

propagation of this pressure wave is much faster ( $>10$ times) than the velocity of blood flow and is related to the stiffness of the blood vessel. ${ }^{3}$ Much as sound waves may be reflected as echoes, pressure waves can be reflected. This happens at sites (for example, branches or tapering) where vessel diameter and/or stiffness (impedance) changes. Reflected pressure waves travel back towards the heart augmenting the forward pressure wave and reducing the forward flow of blood.

In the aorta wave reflection is responsible for the late systolic augmentation of pressure that is often seen in elderly individuals and may impose an additional load on the heart and impair coronary blood flow. The contribution of reflected waves is often estimated non-invasively by measuring the augmentation index (the proportional rise in late systolic pressure) either from the pressure waveform measured in the carotid artery ${ }^{76}$ or after mathematical transformation of the radial artery waveform. ${ }^{\mathrm{w} 7}$ The pattern of pressure augmentation in a particular artery depends on the distance to downstream reflection sites and speed of wave travel. Differences in these account for the complex changes in the early systolic component of the pressure waveform at different sites.

A different sort of pressure wave is evident at the end of systole-a forward travelling expansion wave. This is effectively a wave of suction generated by the left ventricle. This comes about when active myocardial shortening stops, but the aortic valve is still open. For a short period aortic blood flow continues under its own momentum ${ }^{\text {w8 }}$ and left ventricular pressure declines giving rise to the expansion wave. ${ }^{\text {w9 }}$ This phase corresponds to the period of protodiastole described by Wiggers. ${ }^{\text {w10 }}$ The expansion wave slows the flow of blood from the heart and leads to closure of the aortic valves (valve closure is al so accel erated by formation of vortices behind the val ve leaflets during flow deceleration. ${ }^{\text {w11 }}$

\section{HAEMODYNAMICS OF HYPERTENSION}

Patients with hypertension of several years standing (but without target organ damage) have increased PVR (box 4). ${ }^{8}$ The increase in PVR is present in all vascular territories (for example, renal, splanchnic, skeletal muscle, coronary, and pulmonary). Cardiac index and stroke volume are generally normal or reduced, although heart rate may be higher than in normals. ${ }^{8}$ Following exercise the increased PVR is even more evident and cardiac output is typically reduced, largely because of a failure of stroke volume (SV) to increase in response to exercise. Nevertheless, despite the normal cardiac index, modelling studies suggest that abnormal cardiac performance contributes significantly to elevated blood pressure in established hypertension, particularly in subjects with concentric hypertrophy of the heart. ${ }^{\text {4 }}$

In established hypertension there is also a reduction in arterial compliance and a central shift in blood volume, which may be secondary to reduced venous compliance. However, blood volume and extracellular fluid volume are generally found to be normal.

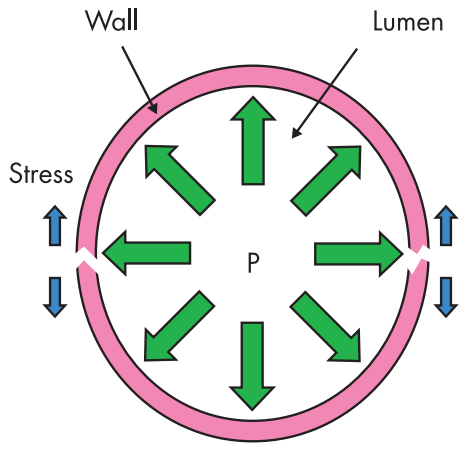

$$
\begin{gathered}
T=P \times R \\
\text { (where } T \text { = tension, } P=\text { pressure, } R=\text { lumen radius) } \\
\sigma=T / h \\
\text { (where } \sigma=\text { stress, } h=\text { wall thickness) }
\end{gathered}
$$

Figure $3 \mathrm{~W}$ all stress and tension in a thin walled tube or chamber (Laplace's law).

\section{HAEMODYNAMICS OF EARLY HYPERTENSION OR PRE-HYPERTEN SIVE STATES}

While the basic haemodynamics of established hypertension are undisputed, there is less agreement about the haemodynamic pattern in young individuals with (comparatively) elevated blood pressures. Since an individual's position within the overall blood pressure distribution tends to remain fairly constant (tracking of blood pressures) these subjects are at high risk of developing hypertension in later life.

In young individuals less than 19 years, most evidence suggests that subjects with the highest blood pressure have increased PVR, though small increases in cardiac index have been seen in some studies. ${ }^{\text {w12 }}{ }^{\text {w13 }}$ There is evidence that young individuals with hypertension have an increased sympathetic activity, though studies in established hypertension are inconclusive. ${ }^{8}$ The question is complicated by methodological difficulties in the assessment of sympathetic nerve activity in vivo and the importance of increased sympathetic activity in the development of hypertension is uncertain. Interestingly, an increased left ventricular mass is a fairly consistent finding in young individuals with raised blood pressure ${ }^{8}$ and is also seen in offspring of hypertensives, ${ }^{114}$ although the functional determinants of this abnormality are not well understood.

In adults (18-40 years) with raised blood pressure (borderline or mild hypertension) PVR is often in the normal range at rest, and the raised blood pressure is attributable to increased cardiac index and heart rate. A prospective study of young males ${ }^{8}$ reported that subjects with high cardiac index/normal total peripheral resistance index pattern changed to a low cardiac index/high resistance pattern at 10 and 20 year follow up; it is therefore plausible that haemodynamic patterns in young individuals with high blood pressure undergo this type of change as they age, accounting for the appearance of the high PVR pattern in established hypertension.

Structural changes in hypertension

Both heart and arteries adapt their structure in response to altered load. This occurs physiologically (for example, during somatic growth) and pathologically in hypertension. Increased pressure exerts an increased load on a thin walled chamber or tube by increasing wall tension according to Laplace's law (fig 3).

M ore complex expressions for wall tension in the heart or blood vessels exist, but this is a reasonable approximation. 


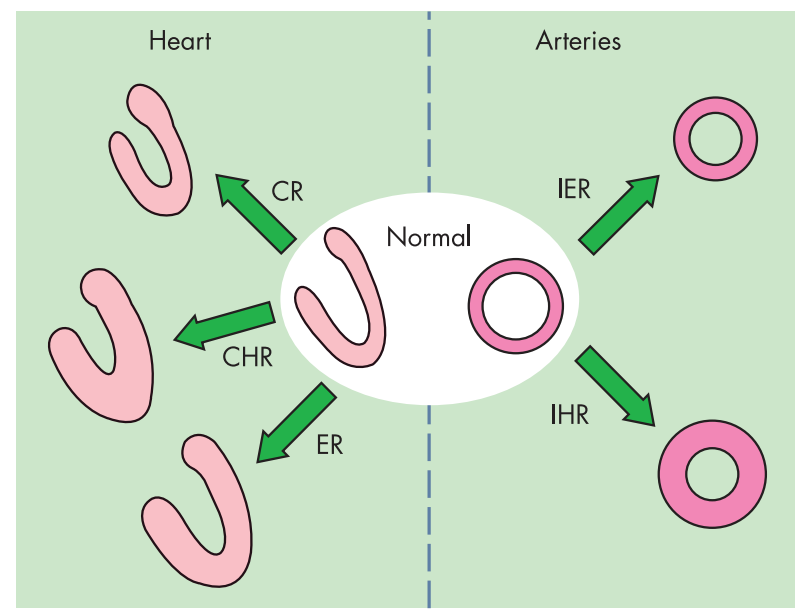

Figure 4 Major patterns of myocardial and vascular remodelling in hypertension. CR, concentric remodelling; $\mathrm{CHR}$, concentric hypertrophy; ER, eccentric remodelling; IER, inward eutrophic remodelling; IHR, inward hypertrophic remodelling. ${ }^{20}$

A rise in tension results in increased wall tensile stress. Normalisation of wall tensile stress can be achieved either by an increase in wall thickness or by a reduction in chamber/ lumen diameter, or both.

Cardiac remodelling in hypertension and its functional consequences

Cardiac structure is influenced by pressure and volume loads. ${ }^{9}$ In hypertension changes in left ventricular structure occur in response to the increased pressure load and represent an attempt by the heart to normalise myocardial wall stress. The increased pressure load in hypertension is primarily caused by the increased resistance, although reduced compliance and possibly altered magnitude and timing of reflected pressure waves make a contribution.

Patterns of remodelling: relation to load Hypertension is associated with a spectrum of structural change in the left ventricle. ${ }^{\text {w15 }}$ The pattern of change may reflect differences between individuals in terms of age, haemodynamics, 24 hour blood pressure profile, arterial stiffness, plasma volume, myocardial performance, neurohormonal status or genetic influences. ${ }^{10}$ w15 $\mathrm{H}$ ypertensive changes can be classified as showing hypertrophy (an increase in left ventricular mass) or remodelling (normal left ventricular mass, abnormal relative wall thickness) (fig 4).

Remodelling is seen in normal aging without hypertension $^{\text {w16 }}$ and is probably an adaptation to preserve ejection fraction despite reduced midwall fibre function. It has become apparent that myocardial fibre shortening is reduced in human hypertension. Early clinical investigations assessed cardiac function using endocardial measurements. Recently, it has been appreciated that there is a discrepancy between shortening measured at the endocardium and at the midwall. $\mathrm{Midwall}$ shortening is commonly reduced in left ventricular hypertrophy and the process of wall hypertrophy allows total wall shortening to remain normal in spite of a depression in fibre shortening - that is, the change in left ventricular geometry allows the chamber function to remain normal. ${ }^{11}$

Normal myocardium contains an interstitial fibrous network upon which the myocytes are arranged. Although hypertrophy primarily involves myocytes, the interstitial network also changes. This occurs initially in a perivascular distribution but progressively extends to cause a widespread interstitial fibrosis. In addition, replacement fibrosis may occur to replace necrotic or apoptotic myocytes. Increased interstitial fibrous tissue is probably important in cardiac dysfunction in hypertension, but the amount of fibrosis is not easy to measure clinically and so differential changes in myocyte hypertrophy and fibrosis cannot easily be assessed in patients.

M ost hypertensives have normal left ventricular structure, but left ventricular hypertrophy predicts a poor prognosis, the almost threefold increased risk being independent of the blood pressure level. ${ }^{12}$ Whether individual remodelling patterns confer additional risk is disputed (Framingham study) and how left ventricular hypertrophy causes increased risk is uncertain. The increase in ventricular arrhythmias ${ }^{13}$ and increased QT duration and QT dispersion seen in left ventricular hypertrophy ${ }^{14}$ may account for the increased risk of sudden death, but other mechanisms such as impaired coronary perfusion could also be important. The electrical abnormalities are likely to be caused by heterogeneous conduction in the ventricle due to increased interstitial fibrosis. There is now increasing evidence that regression of left ventricular hypertrophy with antihypertensive treatment provides cardiovascular protection over and above the reduction in blood pressure levels, ${ }^{15}{ }^{\text {w17 }}$ but again the mechanism of the risk reduction is uncertain.

Consequences of hypertrophy and remodelling Active relaxation is impaired in hypertrophy and remodelling. ${ }^{\text {w18 }}$ The explanation for this is uncertain, al though changes in intracellular calcium handling, ion exchangers, and ion channels are implicated. ${ }^{\text {w19 }}$ Cardiac hypertrophy is al so associated with impaired coronary reserve. This may be caused by a number of mechanisms, including endothelial dysfunction, narrowing of small arteries, microvascular rarefaction, perivascular fibrosis, altered wall mechanics, and relative myocyte hypertrophy. ${ }^{\text {w20 }} \mathrm{A}$ diminished coronary reserve will lead to myocardial ischaemia in the absence of epicardial coronary disease and may further impair relaxation.

Impaired relaxation results in prolongation of isovolumic relaxation time (from aortic valve closure to mitral valve opening) because it takes longer for left ventricular pressure to decrease below atrial pressure. Once the mitral valve is open the slowed relaxation means that left ventricular filling takes longer and there is more blood in the left atrium by the end of the early filling period. This leads to an increased force of atrial contraction. Echo Doppler studies therefore show an early filling wave (E wave), that has a reduced peak velocity and a prolonged duration ( fig $5 \mathrm{~B}$ ) and an increase in the $A$ wave, resulting in a reduced $\mathrm{E} / \mathrm{A}$ ratio. As diastolic dysfunction progresses there is a decrease in left ventricular compliance caused mainly by theincreased interstitial fibrosis. This impairs filling notably. As the heart becomes stiffer, pressure in all the chambers increases. The increased left atrial pressure results in a large pressure gradient in early diastole when the mitral valve first opens, so the peak velocity of the early filling wave is very high. But the reduced ventricular compliance means that pressure increases disproportionately for small changes in volume, so once left ventricular filling begins atrial and ventricular pressures equal ise very quickly, resulting in a very short duration early filling wave ( fig $5 \mathrm{C}$ ).

Mild diastolic dysfunction is often clinically silent; however, if the deterioration of diastolic function is sufficient to significantly reduce ventricular filling and left ventricular end diastolic volume, a reduction in stroke volume will occur and 

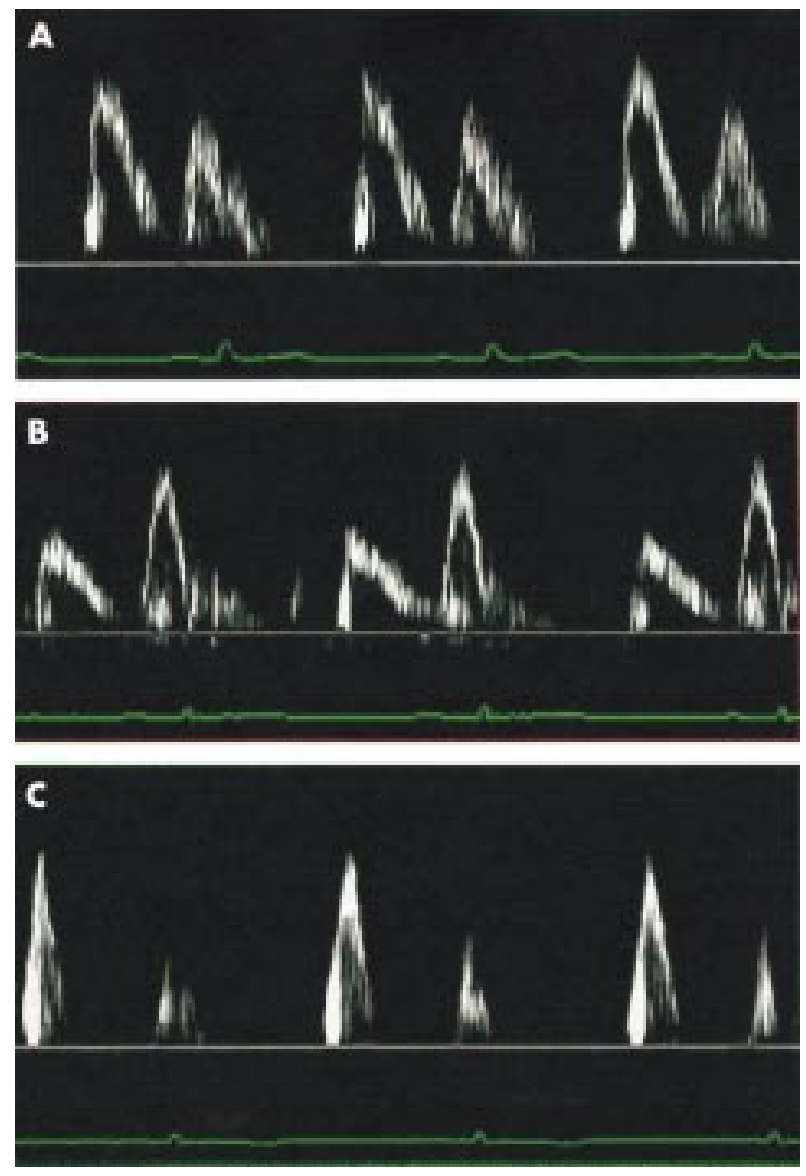

Figure 5 Doppler echocardiographic records showing (A) normal diastolic function, (B) impaired relaxation, and (C) restrictive pattern (that is, severe diastolic function with an increase in left atrial pressure).

patients may develop low output symptoms such as fatigue. As diastolic dysfunction progresses, left ventricular filling pressures become abnormally high and pulmonary congestion may occur. ${ }^{\text {w21 }}$ Thus the symptoms of heart failure may develop in the presence of apparently normal systolic function. Early studies have suggested that over a third of patients with a clinical diagnosis of heart failure have normal left ventricular systolic function ${ }^{\text {w22 }}$; however, it is now becoming apparent that there is considerable overlap between diastolic and systolic dysfunction. ${ }^{16}$ Therefore it is not surprising that diastolic dysfunction associated with symptoms carries an adverse prognosis. $^{17}$

Arterial remodelling in hypertension and its functional consequences

Structural changes in hypertensive vasculature show similarities to those in the heart. In both cases the primary goal appears to be the normalisation of wall stress. The elevated pressure causes an increase in wall tension that is largely experienced by vascular myocytes and the extracellular matrix of the blood vessel. The pattern of remodelling of the vasculature seems highly dependent on the size and function of the artery examined. In part this may reflect the major influence of flow on arterial structure. ${ }^{18}$ Flow affects arteries via the frictional force experienced by the wall as a result of the flow of blood (shear stress). The wall shear stress is sensed by the endothelial cells. In response to shear stress the endothelium releases a number of vasoactive factors that affect arterial tone and growth.
Cardiac and vascular pathology in hypertension: key points

Increased peripheral vascular resistance (PVR) is the hallmark of established hypertension, but altered cardiac function also probably contributes to the raised blood pressure

Large elastic arteries are important in damping the pulsatile flow created by the heart

Pressure wave reflection from downstream affects the pressure waveform in arteries and this is responsible for raised brachial systolic blood pressure compared with central aortic systolic pressure

Deceleration of left ventricular contraction causes an expansion (suction) wave, which is responsible for aortic valve closure

Hypertension causes cardiac and vascular remodelling and hypertrophy. This helps to normalise left ventricular and arterial wall stress and may compensate for a reduction in myocardial fibre function to preserve cardiac output

Hypertensive vessels are not inherently stiffer than normal blood vessels. All vessels become stiffer as they are distended and the increased stiffness in hypertension is a reflection of this

Hypertension is associated with reduced vasodilator reserve and a reduction in microvascular density

Large elastic and muscular arteries

These arteries do not contribute to peripheral vascular resistance, but influence total arterial compliance and wave reflection. The diameter of large elastic arteries such as the aorta or carotid is increased in hypertension. There is also an increase in wall thickness (or at least intima-media thickness (IMT) as measured by ultrasound). ${ }^{19}$ The increase in diameter in these vessels is probably passive; the rise in pressure distends the vessel, while the increased media thickness normalises media stress. In smaller more muscular large arteries, such as the femoral, brachial, and radial, arterial diameter is not increased, although IMT is increased and wall:Iumen ratio is therefore increased.

Numerous studies have reported that arterial stiffness is increased in hypertension. With the possible exception of the carotid artery of young hypertensives, ${ }^{\text {w23 }}$ this increase in stiffness is not caused by a change in the inherent wall properties of arteries (despite the increase in wall thickness) but is a result of the increased distending pressure. Blood vessels do not obey Hooke's law and are non-linearly elastic-that is, they become stiffer when distended. When this is taken into account, the intrinsic elasticity of hypertensive arteries does

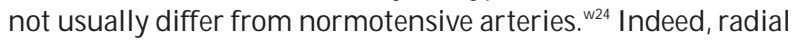
artery stiffness has been reported to be decreased compared with normal when pressure effects are allowed for. ${ }^{\text {w25 Interest- }}$ ingly, these recent findings conflict with the widely held view that hypertensive changes in arterial compliance are essentially an acceleration of the aging process. Aging results in increased arterial stiffness probably through degenerative changes in elastin in the arterial wall. In contrast hypertension does not affect the elastic nature of the arterial wall, although the pressure induced increase in stiffness will worsen age related decreases in arterial compliance. A consequence of the increased arterial stiffness in hypertension is that speed of pressure wave travel will be increased (this is often used as a measure of arterial stiffness). It is also likely that changes between one artery and another (impedance mismatching) result in increased wave reflection and increased pressure augmentation as discussed above. 
Small muscular resistance arteries, arterioles, and the microvasculature

The lumen diameter as a ratio of wall thickness is reduced in small arteries in all forms of hypertension. In essential hypertension this is caused by inward eutrophic remodelling (fig 4), ${ }^{20}$ whereas in some secondary forms of hypertension hypertrophic changes are seen. ${ }^{\text {w26 }}$ This remodelling has a number of consequences beyond normalising media stress. M aximum vasodilation (minimum resistance) is reduced; this results in a reduced vasodilator reserve (most significant in the coronary circulation) and vasomotor responses are enhanced, as a given shortening will induce an exaggerated vasoconstriction. ${ }^{21}$ This effect has been termed the vascular amplifier ${ }^{\text {w27 }}$ and is suggested as a mechanism by which the circulation can chronically maintain elevated resistance without excessive vasoconstriction.

Relatively little is known about arteriolar changes in human hypertension. On the basis of animal studies it has been suggested that arteriolar tone may be increased, but there is little evidence for structural changes in individual arterioles except in accelerated or malignant hypertension. In contrast there is good evidence that the density of the microvasculature is reduced. This can be visualised in a number sites including the retina, sclera, and skin. There is doubt about whether the reduction in microvascular density is "functional" (nonperfused vessels) or structural (obliterated vessels). In our view this is an academic question and in any case it is likely that persistently non-perfused vessels are ultimatel y obliterated. ${ }^{\text {w28 }}$ The consequence of this pruning of the microvascular tree is that resistance is increased (by up to $40 \%$ in some estimates $^{\mathrm{w} 27}$ ) and that maximum tissue perfusion (and consequently nutrient supply) is restricted. This could contribute to the association between hypertension and diabetes (or insulin resistance) as capillary rarefaction in hypertension may result in impaired glucose extraction by skeletal muscle. ${ }^{\text {w29 }}$

\section{CONCLUSIONS}

Hypertension is a disorder of heart and blood vessels. It is a mistake to view these two components in isolation. Abnormalities in arterio-ventricular interaction have been neglected in hypertension research until recently, but may be of critical importance in understanding the development and consequences of this condition.

Authors' affiliations

J Mayet, A Hughes, International Centre for Circulatory Health, 10 th Floor Q EQ M W ing, St Mary's Hospital, and Imperial College Paddington and Clinical Pharmacology, N HL, Imperial College of Science,

Technology \& M edicine, London, UK

\section{REFEREN CES}

1 Messerli FH. This day 50 years ago. N EnglJ Med 1995;332:1038-9.
2 Lever AF, Swales JD. Investigating the hypertensive patient: an overview. In: Swales JD, ed. Textbook of hypertension. 0 xford: Blackw ell Scientific Publications, 1994:1026-30.

3 Fung YC. Biomechanics circulation, 2nd ed. N ew York: Springer, 1997.

4 Milnor WR. Hemodynamics, 2 nd ed. Baltimore: W illiams \& W illiams, 1989

5 Nichols WW, O 'Rourke M F. M CDonald's blood flow in arteries: theoretical, experimental and clinical principles, 4 th ed. London: Edward Arnold, 1998

6 Parker $\mathrm{KH}$, Jones $\mathrm{C}$. Forward and backward running waves in the arteries: a nalysis using the method of characteristics. I Biomech Eng $1990 ; 112: 322-6$

7 Kelly R, Hayward C, Avolio A, et al. N oninvasive determination of age-related changes in the human arterial pulse. Circulation 1989:80:1652-9.

8 Lund-J ohansen P. Haemodynamics of essential hypertension. In: Swales JD, ed. Textbook of hypertension. 0 xford: Blackwell Scientific Publications, $1994 ; 61-76$.

9 Ganau A, Devereux RB, Pickering TG, et al. Relation of left ventricular hemodynamic load and contractile performance to left ventricular mass in hypertension. Circulation 1990;81:25-36.

10 Roman MJ, Ganau A, Saba PS, et al. Impact of arterial stiffening on left ventricular structure. Hypertension 2000;36:489-94

11 Mayet J, Ariff B, W asan B, et al. Improvement in midwall myocardial shortening with regression of left ventricular hypertrophy. Hypertension 2000;36:755-9.

12 Kannel WB. Left ventricular hypertrophy as a risk factor in arterial hypertension. Eur Heart J 1992;13(suppl D):82-8.

c Summarises the Framingham data regarding left ventricular hypertrophy and its associated morbidity and mortality.

13 Mayet J, Shahi M, Poulter N R, et al. Ventricular arrhythmias in hypertension: in which patients do they occur? J Hypertens 1995; 13:269-76

14 Mayet J, Shahi M, McG rath K, et al. Left ventricular hypertrophy and QT dispersion in hypertension. Hypertension 1996;28:791-6.

15 Dahlöf B, Devereux RB, Kjeldsen SE, et al. Cardiovascular morbidity and mortality in the losartan intervention for endpoint reduction in hypertension study (LFE): a randomised trial against atenolol. Lancet 2002;359:1003.

c Suggests that regression of left ventricular hypertrophy reduces cardiovascular events above and beyond blood pressure control.

$16 \mathrm{Yu} \mathrm{CM}$, Lin $\mathrm{H}$, Yang $\mathrm{H}$, et al. Progression of systolic abnormalities in patients with "isolated" diastolic heart failure and diastolic dysfunction. Circulation 2002;105:1195-201.

c Using tissue Doppler echocardiography the authors show that there is considerable overlap between diastolic and systolic dysfunction.

17 Vasan RS, Larson M G, Benjamin EJ, et al. Congestive heart failure in subjects with normal versus reduced left ventricular ejection fraction: prevalence and mortality in a population-based cohort. J Am Coll Cardiol 1999;33:1948-55.

c Shows that heart failure related to diastolic dysfunction has an adverse prognosis although this is not as bad as heart failure related to systolic dysfunction.

18 Langille BL. Arterial remodeling: relation to hemodynamics. Can J Physiol Pharmacol 1996:74:834-41.

19 Hughes AD, Sinclair A-M, G eroulakis G, et al. Structural changes in the heart and carotid arteries associated with hypertension in humans. J Human Hypertens 1993; 7:395-7.

20 Heagerty AM, Aalkjaer C, Bund SJ, et al. Small artery structure in hypertension. Dual processes of remodelling and growth. Hypertension 1993;21:391-7

21 Folkow B. Physiological aspects of primary hypertension. Physiol Rev 1982;62:347-504

c A seminal review of the relation between hypertension and structural changes in the vasculature.

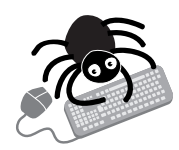

Additional references appear on the Heart website- www .heartinl.com/ supplemental

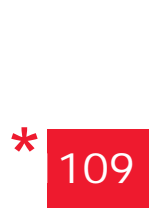

